\title{
The Earth's ionosphere: structure and composition. Dispersive effects, absorption and emission in EM wave propagation
}

\section{Francisco Colomer ${ }^{1}$}

Observatorio Astronómico Nacional

Calle Alfonso XII, 3; E-28014 Madrid, Spain

E-mail: f.colomer@oan.es

Electromagnetic waves, propagating through the Earth's magnetosphere and atmosphere, suffer different effects that need to be understood in order to obtain correct information from cosmic sources. The structure of the ionosphere, its effects on the propagating radio waves, and some techniques to calibrate the received signals, are described.

2nd MCCT-SKADS Training School Radio Astronomy: fundamentals and the new instruments Sigüenza (Spain)

August $26^{\text {th }}-$ September $4^{\text {th }} 2008$

\footnotetext{
1 Speaker
} 


\section{Introduction}

Electromagnetic waves, propagating through the Earth's magnetosphere and atmosphere, suffer different effects that need to be understood in order to obtain correct information from cosmic sources. Figure 1 shows a sketch of the atmosphere structure.

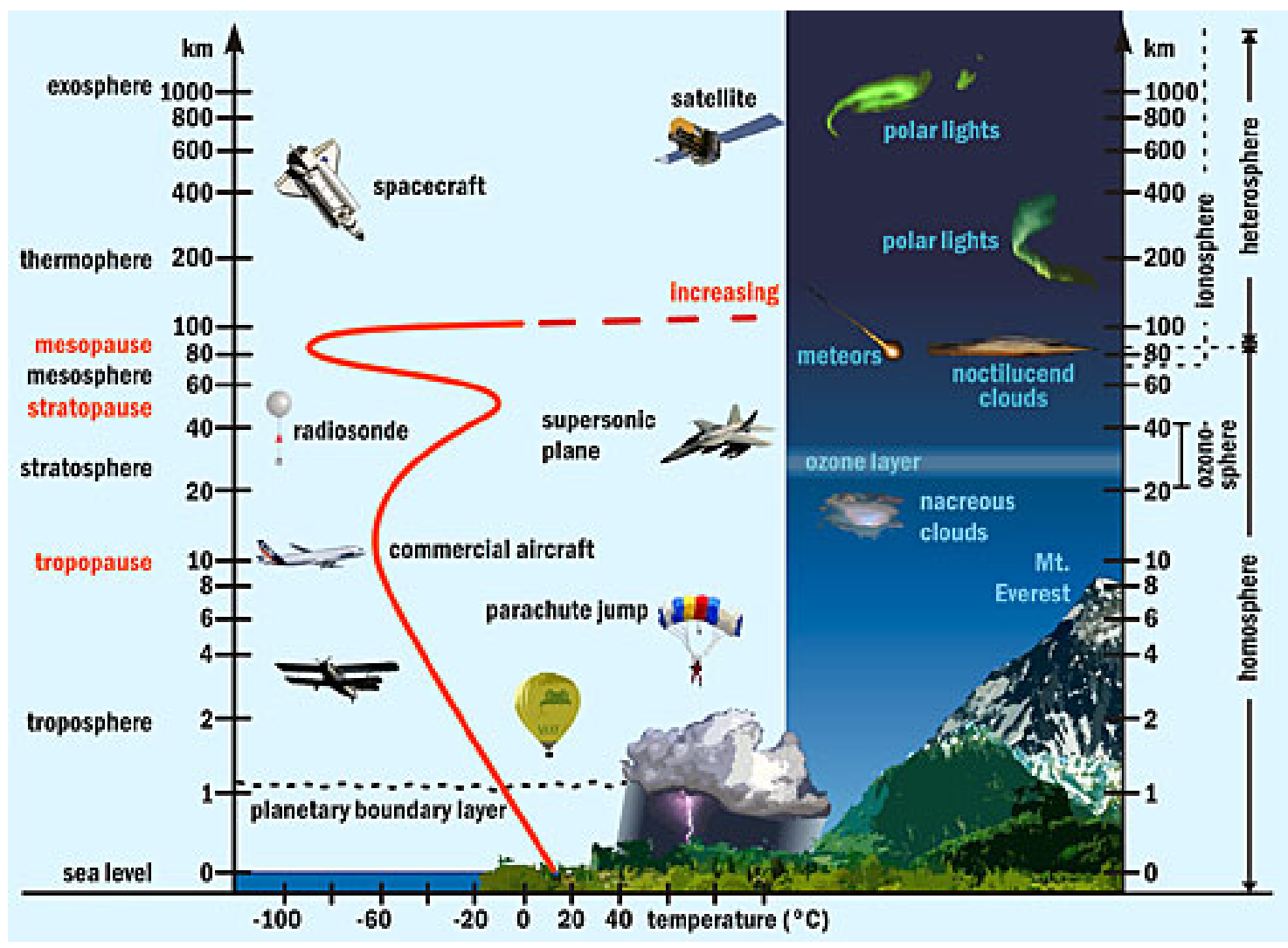

Figure 1: Structure of the Earth’s atmosphere.

Above $50 \mathrm{~km}$ to about $600 \mathrm{~km}$ (370 mi) is the ionosphere, notable for its effects on radio propagation. At these altitudes, atomic oxygen and nitrogen predominate under very low pressure. High-energy solar UV and X-ray radiation ionize these gases, creating a broad region where ions are created in relative abundance. The ionosphere is subdivided into distinctive D, E and $\mathrm{F}$ regions, as solar radiation acts on the different compositions of the atmosphere with height.

- F layer: Known as the Appleton layer, is $120 \mathrm{~km}$ to $400 \mathrm{~km}$ above the surface of the Earth. It is the top most layer of the ionosphere. Here extreme ultraviolet (UV, 10-100 nm) solar radiation ionizes atomic oxygen. It consists of one layer at night, but in the presence of sunlight (during the day), it divides into two layers, labeled F1 and F2. From 1972 to 1975 NASA launched the AEROS and AEROS B satellites to study the F region. 
- E layer: It is the middle layer, $90 \mathrm{~km}$ to $120 \mathrm{~km}$ above the surface of the Earth. Ionization is due to soft X-ray (1-10 nm) and far ultraviolet (UV) solar radiation ionization of molecular oxygen (O2). The Es layer, known as the "sporadic Elayer" it is characterized by small clouds of intense ionization.

- D layer: It is the innermost layer, $50 \mathrm{~km}$ to $90 \mathrm{~km}$ above the surface of the Earth. Ionization here is due to Lyman series-alpha hydrogen radiation at a wavelength of 121.5 nanometer (nm) ionizing nitric oxide (NO).

Gradual refraction in the ionosphere allows radio signals to be propagated long distances. Guglielmo Marconi received the first trans-Atlantic radio signal on December 12, 1901, in St. John's, Newfoundland (Canada) using a $152.4 \mathrm{~m}$ (500 foot) kite-supported antenna for reception. The transmitting station in Poldhu, Cornwall (UK) used a spark-gap transmitter to produce a signal with a frequency of approximately $500 \mathrm{kHz}$ and a power of 100 times more than any radio signal previously produced.

Robert Watson-Watt introduced the term ionosphere in 1926. Edward V. Appleton was awarded a Nobel Prize in 1947 for his confirmation in 1927 of the existence of the ionosphere.

The amount of free electrons caused by ionization affect the propagation of electromagnetic waves through the ionosphere. These effects scale as $v^{-2}$.

Absorption occurs as a result of the interaction between electromagnetic waves and the free electrons in the ionosphere.

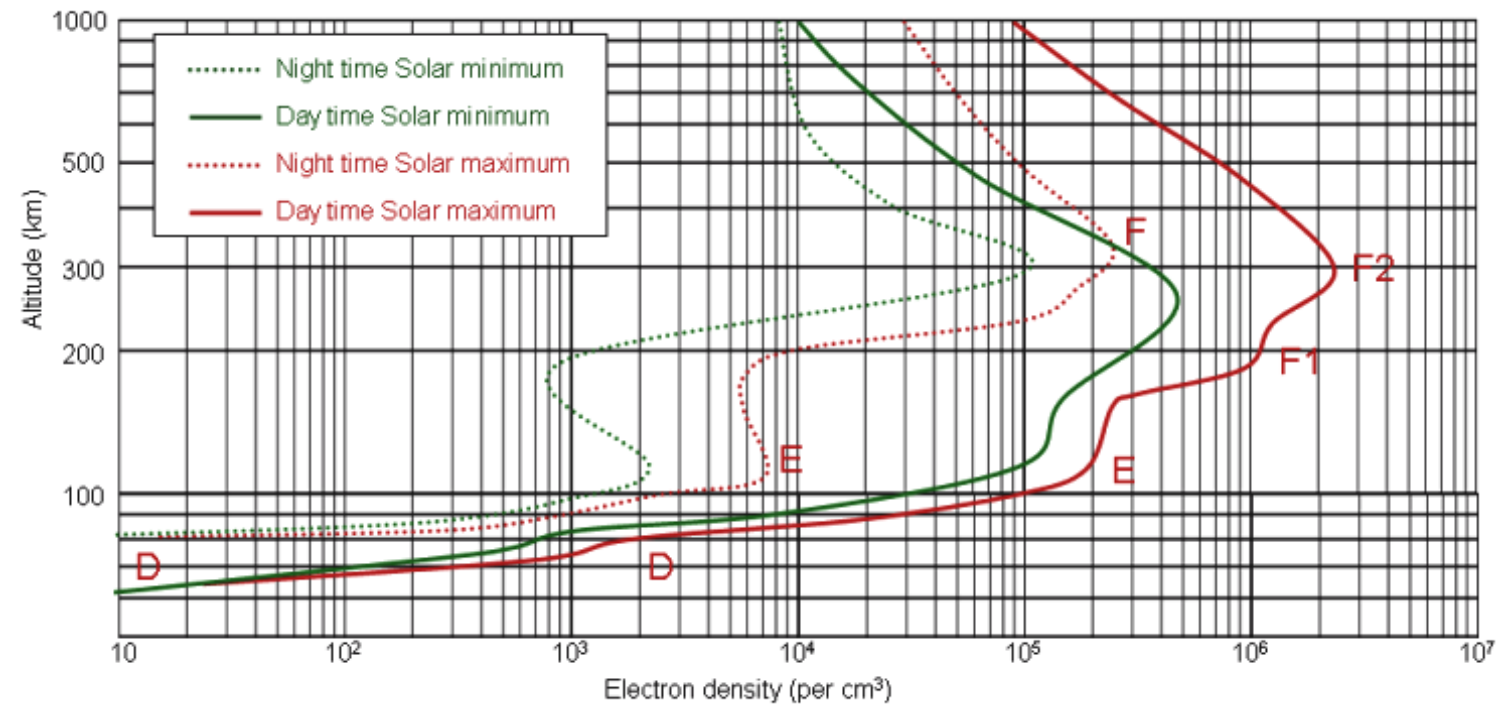

Figure 2: Total Electron Content (TEC) at the Earth’s different ionospheric layers. 


\section{Calibration of ionospheric delay}

\subsection{International Reference Ionosphere}

The International Reference Ionosphere model (IRI), maintained by the scientific community, assumes that each layer is smooth and uniform. Anomalies are known; in reality the ionosphere is a lumpy, cloudy layer with irregular patches of ionization.

Known anomalies are: winter anomaly, equatorial anomaly, equatorial electrojet.

Ionospheric perturbations are due to: X-rays (sudden ionospheric disturbances, SID), protons (polar cap absorption, PCA), geomagnetic storms, or lightning.

\subsection{GNSS}

Data from over 100+ continuously operating dual-frequency GPS receivers in a global network are being used to produce global maps of the ionosphere's total electron content (TEC), replacing ionosondes.

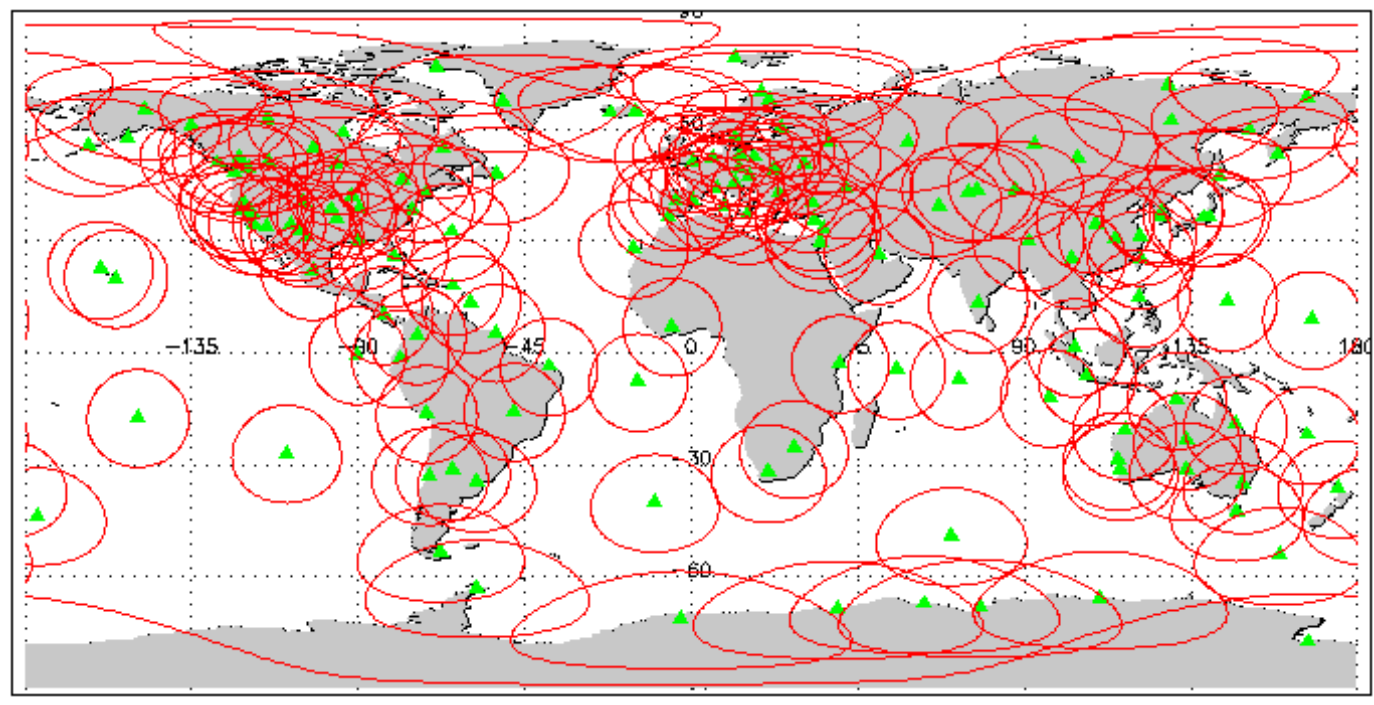

Figure 3: Total Electron Content (TEC) at the Earth’s different ionospheric layers.

\subsection{Widely separated dual frequency observations (S/X)}

Thanks to the dispersive character of the ionosphere, the differential path length effects can be eliminated for unresolved sources (quasars) by making astronomical observations simultaneously at two widely separated frequencies [1]. This is done regularly in geodetic VLBI observations, receiving their radio emission at $8.4 \mathrm{GHz}$ (X band) and $2.3 \mathrm{GHz}$ ( $\mathrm{S}$ band). These effects appear as non-closure phases:

$$
\phi_{c}=\phi_{2}-\left(\frac{v_{1}}{v_{2}}\right) \phi_{1}
$$


The excess path delay can be written as [2]:

$$
\Delta \tau_{\text {group }}^{\text {ion }}=-\left[\left(\tau_{\text {obs }}-\Delta \tau_{S}\right) X \text {-band }-\left(\tau_{\text {obs }}-\Delta \tau_{S}\right) S \text {-band }\right] \frac{v_{S-\text { band }}^{2}}{v_{X \text {-band }}^{2}-v_{S \text {-band }}^{2}}
$$

It is usually assumed that the source position in the sky is the same at both frequencies, which indeed is not always the case (or the source has structure) and this affects the accuracy of the results.

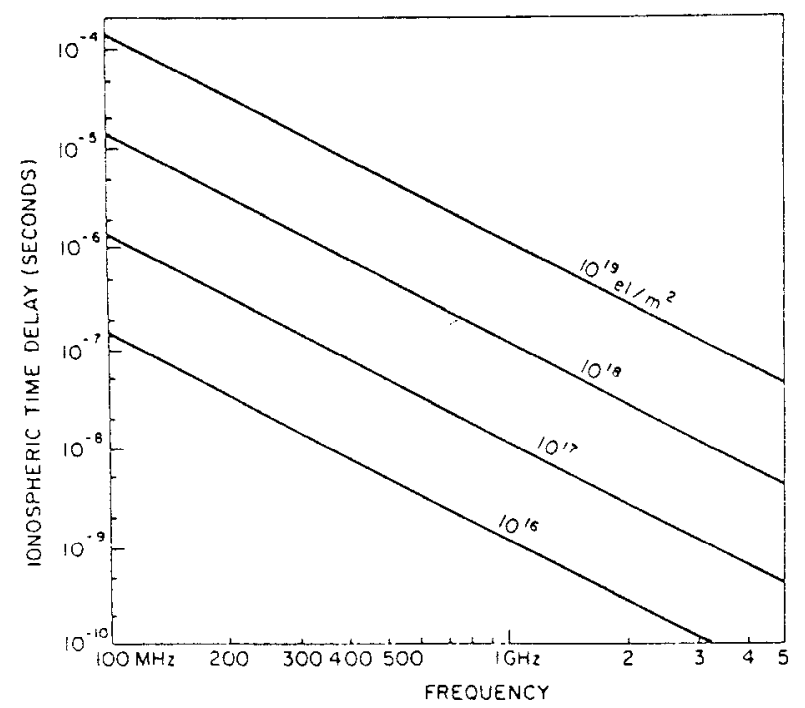

Figure 4: Time-delay versus frequency, for various values of the TEC.

\section{Ionospheric storms}

A sudden ionospheric disturbance (SID) is an abnormally high ionization/plasma density in the $\mathrm{D}$ region of the ionosphere caused by a solar flare.

When a solar flare occurs on the Sun a blast of intense ultraviolet and X-ray radiation hits the dayside of the Earth after a propagation time of about 8 minutes. This high energy radiation is absorbed by atmospheric particles, raising them to excited states and knocking electrons free in the process of photoionization. The low altitude ionospheric layers (D region and $\mathrm{E}$ region) immediately increase in density over the entire dayside. The ionospheric disturbance enhances VLF radio propagation, which can be used to detect solar flares.

The ability to use the GPS network to generate global maps of the ionosphere's total electron content (TEC) in near real-time presents us with a very powerful tool for detecting ionospheric storms and monitoring their behaviour. 


\section{The ionosphere and the SKA}

The joint Argentinean / Brazilian SKA proposal was eliminated because the ionospheric conditions above South America would limit the SKA's performance at low frequencies.

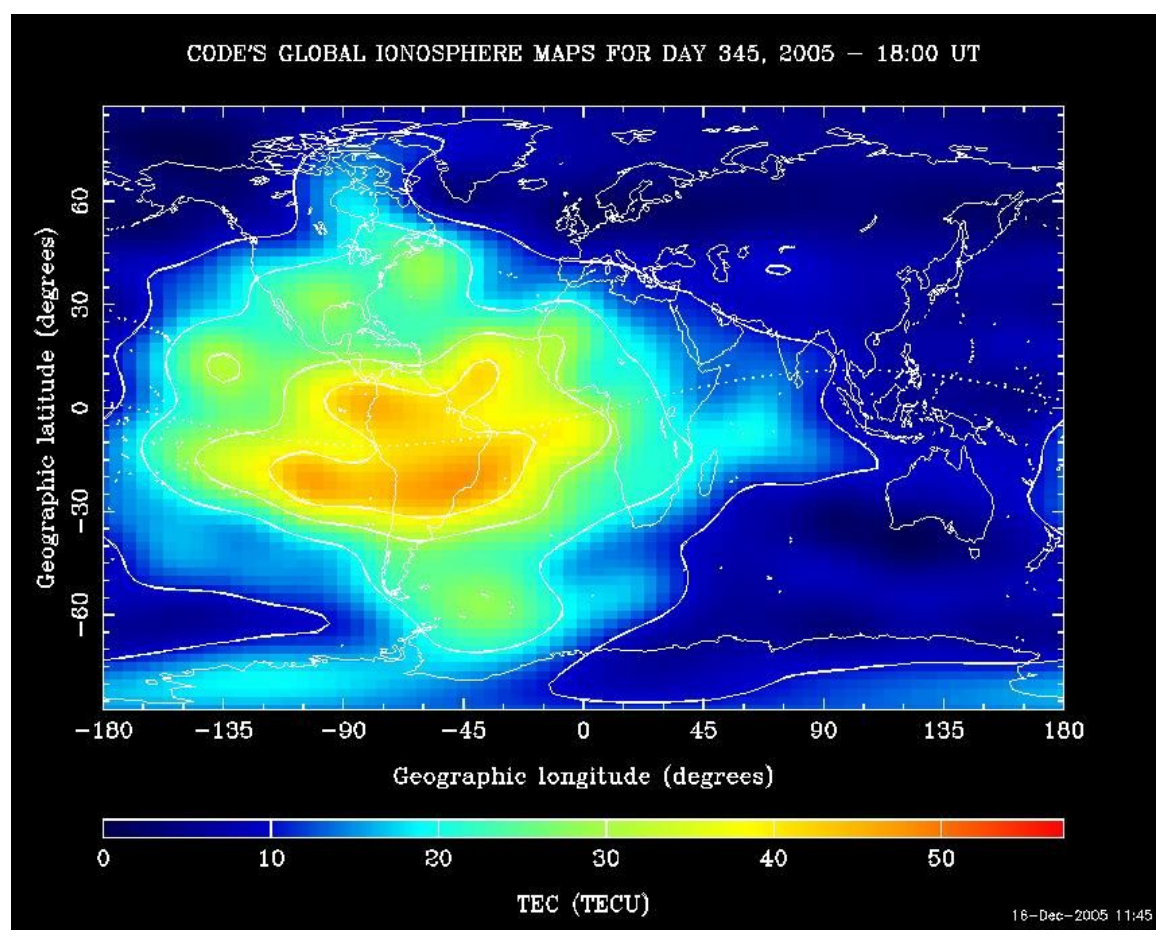

Figure 5: Total Electron Content (TEC) peaks on South America.

\section{Conclusion}

It is important to properly estimate the impact that the ionosphere produces on electromagnetic waves (in particular cosmic radio waves) travelling through it. Several methods are available for such estimation. The dispersive behaviour of the ionosphere is indeed a characteristic to exploit. GPS and VLBI perform observations at two frequencies, to estimate the value of the Total Electron Content (TEC) which, in turn, helps determining the excess path delay.

\section{References}

[1] A. R. Thompson, J. M. Moran, G. W. Swenson, Jr. Interferometry and Synthesis in Radio Astronomy, 2nd Edition. John Wiley \& Sons, Inc. 2001.

[2] B. Rönnäng, Geodesy VLBI observables, in proceedings of the NATO Advanced Study Institute on The Techniques of Very Long Baseline Interferometry. Kluwer Academic Publishers, Series C, Vol. 283, pags. 289 - 302. 1989. 